\title{
On the presumed superiority of analytical solutions over numerical methods
}

\author{
Vincent Ardourel • Julie Jebeile
}

Received: date / Accepted: date

\begin{abstract}
An important task in mathematical sciences is to make quantitative predictions, which is often done via the solution of differential equations. In this paper, we investigate why, to perform this task, scientists sometimes choose to use numerical methods instead of analytical solutions. Via several examples, we argue that the choice for numerical methods can be explained by the fact that, while making quantitative predictions seems at first glance to be facilitated with analytical solutions, this is actually often much easier with numerical methods. Thus we challenge the widely presumed superiority of analytical solutions over numerical methods.
\end{abstract}

Keywords Applied Mathematics · Exactness · Analytical Solutions · Numerical Methods

\section{Introduction}

An important task in mathematical sciences is to make quantitative predictions, which are often done by solving differential equations. ${ }^{1}$ In physics, chemistry or biology, differential equations are used to describe the behavior of target systems. These equations need to be solved to get quantitative predictions, which are expressed with numbers. They are solved either analytically or numerically. Analytical methods can be used to express, when possible, solutions as functions of variables. Then, quantitative predictions are drawn from these

V. Ardourel

Université catholique de Louvain, Institut Supérieure de Philosophie.

J. Jebeile

Science, Normes, Décision (FRE 3593 CNRS, Université Paris-Sorbonne).

1 Quantitative predictions should be understood in a broad sense. They are not limited to predictions about the future states of the target systems. They also include numerical results of the variables that describe a behavior or a property of the target systems. 
analytical solutions. Numerical methods can also be applied to solve equations on computer and provide numerical results. For the purpose of making quantitative predictions, analytical solutions are generally considered the most valuable choice. One of the main reasons is that analytical solutions are exact solutions. This seems to guarantee that the resulting quantitative predictions are accurate. On the other hand, numerical methods generate errors, which may produce results that can deviate too much from the exact solutions.

However, in some cases, scientists use numerical methods even though analytical solutions are available. For example, the Schrödinger equation is generally solved numerically although its analytical solutions are known:

[I]n contemporary research almost all the manipulation of the Schrödinger equation is done not analytically but rather by computers using numerical methods. [...] [S] ome professionals will use computer methods for all problems where accurate wave functions or numerical values of energy are required, even for those special cases for which analytic solutions exist. (French and Taylor, p. 174, we emphasize)

This choice is very surprising. Scientists opt for approximate methods although they actually know the exact solutions. Why do scientists leave out exactness of solutions?

In this paper, we tackle the question why scientists sometimes choose numerical methods rather than analytical solutions in order to make quantitative predictions. While the epistemological consequences of computer simulations in mathematical sciences have been extensively studied in the literature (e.g. Barberousse et al. 2009; Hartmann 1996; Humphreys 2004, 2009; Morisson 2009), the more specific question of how good numerical methods are in making quantitative predictions has not received much philosophical attention until very recently (Fillion and Corless 2014, Fillion and Bangu 2015). Yet answering this question may lead to important claims about applied mathematics.

Most philosophical discussions about mathematics in empirical modelling have centered on the representational function of the mathematical part of models may have. In such a perspective, technical issues related to the application of mathematics might seem of little philosophical interest. However making predictions, confirming or falsifying models crucially depend on how quantitative results are obtained from models. Therefore attention to issues of applied mathematics are crucial and, in particular, it is worth studying how quantitative results are obtained in practice. Going from mathematically formalized theories to quantitative predictions, if possible, is far from being straightforward. It generally requires complex mathematical operations that may introduce approximations and errors, known or unknown, controllable or uncontrollable. In this paper, we make a series of points about the application of mathematics in empirical modelling. By doing so, we aim at questioning the presumed superiority of analytical solutions over numerical methods, and more broadly at investigating scientific practices that form part of the operating knowledge of applied mathematicians. 
In order to explain why scientists sometimes choose to use numerical methods rather than analytical solutions, we rule out the obvious cases for which analytical solutions are not available. This includes cases where the equations are intractable per se, i.e when mathematical results state the impossibility of expressing analytical solutions. This is for example the case of Bruns and Poincaré's results on the impossibility to solve the three-body problem with algebraic functions (Diacu 1996, p. 68). This also includes cases where scientists ignore whether the equations are analytically solvable (see Barberousse and Imbert 2014, p. 267). Instead, we focus on the most problematic cases where analytical solutions are available, but scientists still use numerical methods. Via several examples, we argue that their choice can be explained by the fact that, while making quantitative predictions seems at first glance to be facilitated with analytical solutions, this task is actually often much easier with numerical methods. In other words, ease of making quantitative predictions sometimes prevails over exactness of solutions.

The paper is organized as follows. We first emphasize that analytical solutions are exact solutions whereas numerical methods provide approximate solutions (Section 2). We then outline three main reasons why numerical methods can nevertheless be superior to analytical solutions for the purpose of making quantitative predictions: (i) some analytical solutions make numerical applications difficult or impossible (Section 3); (ii) analytical solutions are sometimes too sophisticated with respect to the problem at stake (Section 4); (iii) analytical methods do not offer a generic approach for solving equations like numerical methods do (Section 5). We finally challenge the presumed superiority of analytical solutions in mathematical sciences (Section 6).

\section{Analytical solutions and numerical methods}

One of the main reasons why analytical solutions are commonly considered superior to numerical methods is that analytical solutions are exact solutions while numerical methods yield approximate solutions. In this section, we first make clear the distinction between exact and approximate solutions (Section 2.1 ) before investigating the notion of "analytical solution" (Section 2.2). We then discuss in what sense numerical methods yield approximate solutions (Section 2.3).

\subsection{Exact and approximate solutions}

Let us begin to make clear the notion of exact solution by contrasting it with the notion of approximate solution. An exact solution $x$ of an equation $E$ can be defined as a mathematical object that makes true the equation $E$. For example, the equation " $d x / d t=x "$ is true if $x$ refers to the exponential function $x(t)=e^{t}$. As a consequence, a solution is exact if, put it into the equation, identity is obtained. If the function $x(t)=e^{t}$ is put into the equation $d x / d t=x$, the identity $1=1$ is obtained, meaning that it is its exact solution. 
By contrast, an approximate solution is a mathematical object that does not make true the equation $E$. The difference between an exact and an approximate solution can be expressed with a notion of error. Let us consider $y(t)$ an approximate solution of the equation $E$ and $x(t)$ its exact solution that are two functions defined within a domain of values $D$. In this case, $y$ is an approximate solution in the sense that the error $e(t)=|x-y|$ is non-zero for at least one value of the variable $t$ within the domain $D$. However, in order that $y$ be considered as an approximate solution, and not only an arbitrary function, the error has to be small enough compared with the exact solution. An acceptable approximate solution will depend on the modelling context. But, if the error is too large, it seems there is no sense in considering $y$ as an approximate solution of the equation. For example, $y(t)=1+t+t^{2} / 2$ can be viewed as an approximate solution of the equation $d x / d t=x$ when $t$ is close to zero but not if $t$ is too large. ${ }^{2}$

With this distinction in mind between an exact and an approximate solution, let us now turn to the difference between analytical solutions and numerical methods used to solve differential equations.

\subsection{Analytical solutions}

Analytical solutions are few but important in mathematical sciences. For example, well-known analytical solutions in physics are the solutions of the harmonic oscillator in classical mechanics, the Poiseuille equation in hydrodynamics, and the Schrödinger equation for a particle in an infinite potential well in quantum mechanics. However, there is no detailed discussions about what an "analytical solution" is. Following Humphreys, analytical solutions are exact solutions which are expressed with closed-forms or infinite series (2004, p. 64). The aim of this section is to explicate this definition.

First, let us make clear that there are different forms of analytical solutions, i.e closed-forms and infinite series. A solution expressed as an infinite series corresponds to an infinite sum of functions with the form $y(x)=\sum^{\infty} c_{k} f^{(k)}(x)$ where $f(x)$ is a function - e.g $x$ or $\cos (x)-c_{k}$ are algebraic coefficients, and the symbol " $(k) "$ corresponds, for example, to the $k^{t h}$ derivative or the $k^{t h}$ power. In order to be meaningful, infinite series have to be convergent, which means that, once applied to a particular value of $x$, the series leads to a finite number. However, the blind spot in this definition is the function $f$, for which we suggest that it has to be expressed as a closed-form.

The notion of closed-form function is more controversial (Borwein and Crandall 2013, p. 50-51). Closed-form functions are expressed with a finite number of algebraic operations from a given accepted set of functions, such as rational functions (i.e fractions of polynomial functions). However different kinds of closed-form functions can be distinguished depending on the accepted algebraic operations. Thus closed-form functions can be algebraic functions,

2 This is justified by the fact that $y(t)$ is the first terms of Taylor series of the exponential function at $t=0$. 
elementary functions, liouvillian functions, and special functions. ${ }^{3}$ For example, $x(t)=e^{t}$ is an elementary function, which is the solution to the differential equations $d x / d t=x$, and $x(t)=J_{0}(t)$ is a special function, which is a Bessel function of the first kind of order zero and the solution to the differential equation $t^{2} d^{2} x / d t^{2}+t d x / d t+t^{2} x=0$.

It has to be stressed that the frontier between closed-form functions and infinite series is somewhat arbitrary. As Eric Weisstein points out "the choice of what to call closed-form and what not is rather arbitrary since a new "closedform" function could simply be defined in terms of the infinite sum" (quoted in Borwein and Crandall 2013, p. 50). In other words, closed-form functions can be seen as just a proper subset of infinite series. But, although closedform functions can be rewritten as convergent infinite series (like the special function $J_{0}(t)$ ), it is still unclear why some infinite series are classified by mathematicians as not having a possible corresponding closed-form. There are indeed some infinite series that are generally considered as not being closedform functions. In Section 3.3, we will discuss such an example (the solution of the N-body problem), from which we will argue that the often presumed superiority of analytical solutions over numerical methods has to be mitigated.

Secondly, analytical solutions are exact solutions in the sense previously discussed (Section 2.1): they are mathematical objects that make true the equation. We point out that there are different ways to find exact solutions. The exact solutions can be obtained in following mathematical transformations to solve the equation. Even if there is no general recipe for solving a given differential equation, there are different techniques to derive the solutions depending on the form of differential equations (like the method of "separation of variables" or the use of canonical forms for some linear differential equations). A solution can also be found by accident. Whatever the way they are found, analytical solutions are exact solutions, and, because of it, analytical solutions are generally considered as the best option for getting quantitative predictions. This is a central reason why analytical solutions seem to be the best option for getting quantitative predictions, especially as numerical methods are approximate, as we will now see.

\footnotetext{
3 Algebraic functions are built from rational functions with the following operations: addition, subtraction, multiplication, division, and exponentiation with integral and fractional exponents. Elementary functions are built in admitting in addition the operations of exponentiation in general and derivation. Liouvillian functions admit in addition the operation of integration (Goriely 2001, pp. 38-40 and Zoladek 1998, pp 2-3). Closed-form functions admit in addition special functions like the non-liouvillian Airy and Bessel functions. Special functions are functions that are purposely defined as the solutions of some differential equations. For a detailed discussion on the role of special functions in physics, see (Batterman 2007). We borrow this list from Fillion and Bangu (2015, p. 4) to which we add liouvillian functions (Singer 1990, p. 66). Liouvillian functions play indeed an important role in classical mechanics: they are the solutions of integrable systems like the Kepler problem or the one-dimension simple pendulum (Babelon et al. 2003, chap. 2).
} 
2.3 Numerical methods

There are numerous numerical methods used to solve differential equations. The most famous methods may be the Euler methods and the Runge-Kutta methods. From a general point of view, numerical methods can be classified into two main families: one-step methods and multi-steps methods (Hairer et al. 1992, Ortega 1992 among others). The aim of this section is to make clear in what sense numerical methods are approximate. More generally, this section may also be a first attempt to remedy the lack of philosophical discussion about numerical methods in mathematical sciences.

Numerical methods are methods that transform differential equations into difference equations. They provide a recursive algorithm for making calculation that is then carried out on a computer. ${ }^{4}$ Let us illustrate this by introducing a simple one-step method: the forward Euler method. Let us consider the ordinary differential equation $d x / d t=f(x, t)$ with the initial condition $x_{0}$. The forward Euler method leads to the following difference equation $\left(x_{k+1}-\right.$ $\left.x_{k}\right) / h=f\left(x_{k}, t_{k}\right)$. The derivative $d x / d t$ is approximated by the ratio $\left(x_{k+1}-\right.$ $\left.x_{k}\right) / h$, and the variables $x$ and $t$ of the function $f(x, t)$ are both evaluated at the step $k$. This numerical method thus defines the series $\left(x_{k}\right)$ following the recursive rule $x_{k+1}=h f\left(x_{k}, t_{k}\right)+x_{k}$ where $h$ is a discrete time step and $t_{k}=h k$. For example, starting from the initial condition $x_{0}$, one has $x_{1}=h f\left(x_{0}, t_{0}\right)+x_{0}$. Then, $x_{2}=h f\left(x_{1}, t_{1}\right)+x_{1}$, and so on until the last term of the series $x_{N}=h f\left(x_{N-1}, t_{N-1}\right)+x_{N-1}$.

Let us now introduce another series $\left(x\left(t_{k}\right)\right)$ for which $x(t)$ is the exact solution to the previous differential equation. This series corresponds to the sampling of the exact solution at the $N$ instants $t_{1}, t_{2}, \ldots t_{k}, \ldots t_{N}$. This series, even discrete, is exact in the sense that each term $x\left(t_{k}\right)$ is a certain point of the exact function $x(t)$. Under these conditions, one can state that the numerical method is approximate since the series $\left(x_{k}\right)$ and $\left(x\left(t_{k}\right)\right)$ are not identical. In other words, for each $k$, one has in general $x_{k} \neq x\left(t_{k}\right)$. One can thus define an error for each step - often called consistency error or local error - that is $\left|x\left(t_{k}\right)-x_{k}\right|$ and a global error $\max _{1<k \leq N}\left|x\left(t_{k}\right)-x_{k}\right|$, which depend on time step $h$. This shows in what sense numerical methods are approximate: they lead to discrete series that differ from the discrete sampling of the exact solution. In addition, we shall emphasize that the function $f$ has generally to be approximated by another function that can be computed in a finite number of operations. For example, the function $f$ can be replaced by its truncated Taylor series, which leads to an additional source of error called truncation

\footnotetext{
4 We emphasize that these algorithms are not doomed to be implemented on a computer since numerical methods (e.g Euler methods) have been used long before the development of computers. Numerical calculations were made by hand. However, in this paper, we are interested in the current use of numerical methods in mathematical sciences, which is based on computers.
} 
error. As we have seen, numerical methods are approximate before even being implemented on a computer. ${ }^{5}$

In Section 2, we have seen that analytical solutions are exact whereas numerical methods yield approximate solutions. However, as the quotation from French and Taylor (in the Introduction) illustrates, scientists sometimes choose to use numerical methods rather than analytical solutions. This is very surprising since, here, scientists make the choice to sacrifice exactness. In the remainder of the paper, we explain this choice: we argue that numerical methods provide quantitative predictions more easily than analytical solutions. In other words, ease of making quantitative predictions sometimes prevails over exactness of solutions. The first reason that we will now present is that some analytical solutions make numerical application difficult or impossible.

\section{Risk of cumulative errors}

At first glance, we might think that numerical methods are not as good as analytical solutions in yielding quantitative predictions. Numerical computations generate errors that can accumulate and thereby can deviate too much from the theoretically exact values. Analytical solutions seem to avoid such a risk in that they are ready-to-use solutions: it is commonly thought that, once they have been expressed, one has in principle only to replace the parameters and the variables of the solutions with numerical values in order to obtain quantitative predictions. However this view is misleading. In this section we show that, like numerical computations, numerical application with analytical solutions sometimes encounters the risk of accumulating errors. Thus, we argue that numerical methods can be preferable when the risk is higher in analytical methods.

We first present the risk of accumulating errors in numerical computations (Section 3.1) and we explain why this risk is also present in numerical application with analytical solutions (Section 3.2). Then we present cases for which there might be no hope to overcome this risk with analytical solutions and the only remaining option is to use numerical methods for making quantitative predictions (Section 3.3).

\subsection{Error accumulations in numerical computations}

We have contented (Section 2.3) that numerical methods, before even being implemented on a computer, are approximate. We now show that, once implemented, the corresponding numerical computations generate errors that can make quantitative predictions inaccurate. These errors are round-off errors that are generated at each step of the numerical computations and which can accumulate.

\footnotetext{
5 Additional errors are produced all along numerical computations though, as we will see in Section 3.
} 
In order to see this, let us introduce the series $\left(\tilde{x}_{k}\right)$. It differs from both previous series $\left(x\left(t_{k}\right)\right)$ and $\left(x_{k}\right)$ in that it corresponds to the computed values at each step of the numerical computations. For example, let us consider the previous Euler method, and let 0.1 be the value of the discrete time step $\tilde{h}$ and $\tilde{x}_{0}=9.7$ the initial condition. The first step in this series is obtained from the numerical computation: $\tilde{x}_{1}=\tilde{h} f\left(\tilde{x}_{1}, \tilde{t}_{1}\right)+\tilde{x}_{0}=0.1 \times f(9.7,0.1)+9.7$. One can then compute $\tilde{x}_{2}, \tilde{x}_{3}$, etc. At each step, $\tilde{x}_{k}$ differs from the theoretical value of the variable $x_{k}$ in the series $\left(x_{k}\right)$ obtained from the numerical methods; this difference $\left|\tilde{x}_{k}-x_{k}\right|$ corresponds to the round-off errors generated by numerical computation. Finally, the error that scientists are interested in is $e_{k}=\left|\tilde{x}_{k}-x\left(t_{k}\right)\right|$ between the computed value and the exact solution of the differential equation.

At each step $k$, the errors $e_{k}$ accumulate. If the sum of these errors becomes high, the numerical results would deviate too much from the exact solution, therefore producing numerical artefacts. For instance, this can be the case of the equations of motion for conservative mechanical systems. Because errors accumulate, the numerical computations of these equations can lead to artificial dissipation or artificial forcing, which is in contradiction with energy conservation. Let us consider the case of the simple pendulum, which is described by the differential equation $d^{2} \theta / d t^{2}=-g / l \sin (\theta)$. Stern and Desbrun (2008) showed that numerical instabilities occur when the forward Euler method is used, leading to artificial forcing. Although the theoretical trajectories in the phase-space are closed curves - as the system is conservative - the numerical trajectories are increasing spirals, which correspond to an artificial forcing (Figure 1, on the left).

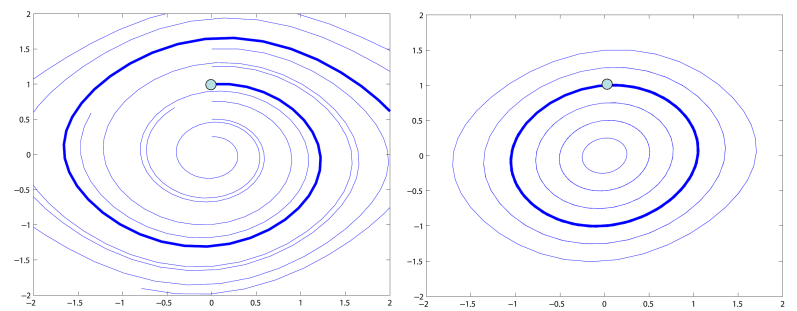

Fig. 1 Numerical computations of the simple pendulum equation with forward Euler method (on the left) and symplectic Euler method (on the right). On the left, the trajectories are increasing spirals because of numerical instabilities. On the right, the trajectories are closed curves, in agreement with the theoretical behaviour of conservative systems (Stern and Desbrun 2008).

Even if error accumulation in numerical computations is a risk, we would like to stress that numerical computations are not doomed to be useless. On the one hand, there are many cases where error accumulation does not occur. For instance, the numerical computations of the simple pendulum with the symplectic Euler method lead to closed trajectories in the phase space (Figure 
1 , on the right). On the other hand, even if there is artificial forcing due to cumulative errors, this artificial forcing may be small enough for the predictions to still be useful.

One could think that error accumulation is specific to numerical computations. In what follows, we want to argue that this risk also concerns numerical applications with analytical solutions.

\subsection{Generated errors with numerical applications}

One might think that numerical applications are easily derived from analytical solutions. It is indeed commonly thought that, once analytical solutions have been expressed, one has in principle only to replace the parameters and the variables of the solutions with numerical values in order to obtain quantitative predictions. Thus, the error between the exact solution and its calculated value $|x(t)-\tilde{x}(t)|$ could be arbitrarily small depending on the number of decimals. For example, if $x(t)=e^{t}$, then $x(1) \approx 2.718$, or $x(1) \approx 2.718281$, or $x(1) \approx$ 2.718281828459 depending on where truncation occurs.

However this view is misleading. The fact that analytical solutions are ready-to-use does not imply that one can easily derive numbers from them. The physicist T. L. Einstein suggests that having analytical solutions is not all of it:

While the discovery of exact solutions is always intellectually exciting and captivating, they are often of little use in confronting experiments - physical or numerical - unless the formulas are numerically tractable.

Thus, even when a problem is formally solved, neither theoreticians or funding agencies should view it as completed until the formalism can be rendered in a way that allows the extraction of numbers, even if approximately. (Einstein 2003, p. 820)

As this quotation suggests, another difficulty arises with analytical solutions, which concerns what Einstein here calls the "extraction of numbers". This is the process of going from the analytical solutions to the quantitative predictions. This process is rarely a mere and direct replacement of the parameters and the variables in the solutions with numerical values, as commonly thought. Instead, this process sometimes involves doing several numerical operations, each of them generating round-off errors.

Having an analytical solution to a differential equations does not guarantee accurate quantitative predictions. As Fillion and Bangu (2015) claim, "contrary to a persistent myth, [...] having an exact solution is not generally more epistemologically beneficial than lacking one" (abstract). This is because the numerical application, from the analytical solution to the numerical value, may require numerous numerical computations that all generate errors, and the summation of these errors may accumulate. The "myth" is actually a misleading generalization of the wide use of elementary functions (e.g rational functions, exponential functions, sine functions). However, for other functions, numerical application does not always lead to accurate numerical results: 
When there is an exact solution, but no elementary solution, it is necessary to rely in some way on infinite series representation of the solution to evaluate it at some time. With respect to calculations, the difficulty with infinite series representations is that we cannot sum an infinite number of terms. It then seems that we can evaluate the solution to arbitrary accuracy by using increasingly long (but finite) truncated series. An interesting situation arises when we have a perfectly good analytic solution in the form a uniformly convergent Taylor series, which converges so slowly that it ends up being of no practical use for computation. (Fillion and Bangu 2015, p. 6)

Infinite series are not always convenient for numerical application. The reason, as Fillion and Bangu claim, is that one cannot sum an infinite number of terms. In such a case, one can only sum the terms of a truncated version of the series. But such finite summation may lead to an inaccurate numerical result. Fillion and Bangu (2015) illustrate this with the Taylor series of the Airy function (a special function). They write "[n] umerically, even if the series converges for all $x$, it might be of little practical use, since the theoretical uniform convergence might not translate to success in numerical contexts" (p. 6). $A i(-t)$ is the analytical solution to $d^{2} x / d t^{2}=-t x$. The equation describes an harmonic oscillator whose stiffness increases over time. Fillion and Corless (2014) insist that, although the infinite Taylor series of Airy function "converges uniformly, the floating-point computation diverges" (p. 1461). This means that a numerical computation of the Taylor series of the function - e.g. $A i(-12.82)$ - with floating-point arithmetic does not necessary tend to the exact value while the numerical computation include more and more terms of the series. ${ }^{6}$ Such a numerical computation thus seems to be useless for the purpose of making quantitative predictions.

Another example is given by Forsythe (1970, p. 934), which is quoted by Corless and Fillion $\left(2014\right.$, p. 14). It is shown that the calculation of $e^{-5.5}$ based on Taylor series is not numerically accurate. More precisely, the use of the Taylor series - i.e $x(t)=\sum_{0}^{\infty} t^{n} / n$ ! - to evaluate the number $e^{-5.5}$ in a floating-point system with a 5 -digit precision leads to an inaccurate numerical result. Summing up the first twenty-five terms of the corresponding Taylor series gives a number with $37 \%$ relative error, which is obviously too high to be useful. Nevertheless there are in this case other ways to evaluate $e^{-5.5}$. For instance, the inverse function can be used to give a better approximation of the value of $e^{-5.5}$ : one computes $e^{5.5}$ and then the inverse of it. Thus, in this case, the numerical issue can be overcome by using mathematical properties of the exponential function, which is possible since it is a closed-form function, and more precisely an elementary function.

Thus, making quantitative predictions with analytical solutions might be not so different than with numerical methods, from both epistemic and method-

\footnotetext{
6 This phenomenon is an example of a catastrophic cancellation. This series is indeed an alternating series in which differences between big numbers with finite significant figures are used to evaluate small numbers. For details, see (Corless and Fillion 2014, p. 15).
} 
ological points of view. Numerical applications with analytical solutions also generate errors that can occur and thus make quantitative predictions inaccurate. Nevertheless, the parallel between applying analytical solutions and numerical methods should not be overstated. There are differences between both ways of making numerical applications.

First, unlike analytical solutions, round-off errors in numerical computations occur over the evolution time of the system. The reason is that, in order to calculate numerically $\tilde{x}(t)$, the calculated numerical values of previous points, e.g. $\tilde{x}(t-1), \tilde{x}(t-2), \tilde{x}(t-3) \ldots$, are required. In other words, long-term predictions are less accurate than short-term ones, and the approximated trajectory can deviate arbitrarily far from the true one. For analytical solutions, this does not happen because the calculation of $\tilde{x}(t)$ with analytical solutions is not based on calculated values of the previous points (each term of the series used to calculate $\tilde{x}(t)$ deals with functions of the same instant $t$ ). Provided that the analytical solution offers a means of calculating each trajectory point with arbitrary accuracy (independent of the accuracy of other points), longterm predictions can be made just as accurate as short-term ones, so that the calculated trajectory stays close to the true one. ${ }^{7}$

There is a second difference between numerical applications with analytical solutions and numerical methods. In the case of analytical solutions, there is sometimes the option to express infinite series with closed-form solutions and, in this way, to overcome the problem of generating errors. For calculating $e^{-5.5}$, one can overcome this problem by using the inverse function. Similarly, one can suppose that scientists could use the Airy function without using its Taylor series. Thus, there might be hope to make quantitative predictions from the closed-form version of the Airy function.

However, in what follows, we present cases for which numerical application is impossible: some analytical solutions are only expressed with slowly convergent infinite series and thus make impossible to avoid the problem of generating errors in numerical applications.

\subsection{Impossible numerical applications}

Here we present a case where having the analytical solution does not help at all in making quantitative predictions and numerical methods are used instead. Such a case is exemplified by the N-body problem of celestial mechanics that we now discuss.

In the N-body problem of celestial mechanics, $N$ particles interact with each other gravitationally. $m_{i}, r_{i}$ are the mass and the position of the $i^{t h}$ particle, respectively, and $t$ is time. The equations of motion are:

$$
m_{i} d^{2} r_{i} / d t^{2}=\sum_{i \neq j} G m_{i} m_{j}\left(r_{i}-r_{j}\right) /\left|r_{i}-r_{j}\right|^{3}
$$

\footnotetext{
7 We are in debt with one of our anonymous reviewers for making this clear.
} 
There are analytical solutions to the N-body problem (Sundman 1907, 1909; Wang 1991), which are perfectly general and have been viewed as theoretical successes. However, these solutions remain useless for physicists: neither Sundman's solution nor Wang's solution can be used to compute the trajectories of an N-body system. This failure is intrinsically due to the form of these solutions, which are infinite convergent series in powers of $t^{1 / 3}$, and thus have no practical use to make quantitative predictions. As Wang himself writes:

Although the conclusion given here provides a way to integrate the Nbody problem, one does not obtain a useful solution in series expansion.

The reason for this is because the speed of convergence of the resulting solution is terribly slow. One has to sum, for example, an incredible number of terms, even for an approximate solution of first order in q, p, t. (Wang 1991, p. 87) ${ }^{8}$

Sundman's solution and Wang's solution are infinite series that are mathematically perfectly acceptable in that they are not divergent series. But the reason for the failure of these solutions is that they both converge too slowly. Slow convergence makes it impossible to obtain quantitative predictions from the solutions. In order to make quantitative predictions from the infinite series, the sum has to be truncated and only the first terms of the series are summed. However, if the speed of convergence is too low, summing the first terms leads to inaccurate numerical results. To get a sufficiently good approximation from slowly convergent infinite series, it would require summing a considerable number of terms, which is not possible in practice. As Florin Diacu asserts about Sundman's solution and Wang's solution:

One would have to sum up millions of terms to determine the motion of the particles for insignificantly short intervals of time. The round-off errors make these series unusable in numerical work. From the theoretical point of view, these solutions add nothing to what was previously known about the n-body problem. (1996, p. 70).

In other words, in the case of Sundman's and Wang's solutions, slow convergence makes these infinite series useless since summing up millions of terms would only give satisfying quantitative predictions for very short periods of time. That is why the available analytical solution here is useless for the purpose of making quantitative predictions. More than that, it seems to make numerical application impossible because, contrary to the case of the Airy function, one does not know any closed-form solution to the N-body problem (for $N>2$ ). The problem does not admit elementary solutions, and more generally does not have liouvillian solutions because it does not contain conserved quantities enough for the equation to be solved under some mathematical transformations that are action-angle variables (Babelon et al. 2003, p. 10; Masoliver and Ros 2011) ${ }^{9}$. Thus one cannot overcome the problem of generating errors. That is why, in order to study the trajectories of N-body systems,

$8 q, p$ are coordinates of phase space and $t$ is time.

9 Action-angles variables are conjugate variables $(I, \theta)$ - sometimes written $(J, \omega)-$ such as the action variables $I(J)$ are constant with time (Babelon et al. 2003, p. 10; Goldstein 
scientists have to use numerical methods. According to Malte Henkel, "the speed of convergence [of Sundman's series] is so low that a mere numerical computation with numerical methods directly applied to (eq. 1) [i.e. the equations of motion], without using infinite series, is more reliable" (2001, p. 175). Despite numerical errors occurring with numerical methods, they are much more reliable than analytical solutions to obtain the trajectories of bodies.

From a general point of view, speed of convergence is a key property of infinite series since, depending on this speed, solutions can or cannot be used to make quantitative predictions. Infinite series solutions with slow convergence cannot be used to provide sufficiently accurate numerical results. In such a case, numerical methods are more valuable than analytical solutions in order to make quantitative predictions. There are other reasons to choose numerical methods rather than analytical solutions. As we will now argue, analytical solutions can be inadequate for the type of problem to which they are the exact solutions. More precisely, analytical solutions may require mathematical techniques that are too sophisticated to be appropriate for the problem at stake.

\section{Excessively sophisticated analytical solutions}

Analytical solutions are sometimes an excessively sophisticated mathematical machinery for the problem at stake. In this case, analytical solutions, although they are available, are inadequate for the type of problem to which they are the exact solutions. In order to illustrate this, let us go back to the example of the simple pendulum, which is one of the simplest model in classical mechanics.

The simple pendulum is composed of a mass point (with mass $m$ ) attached to a massless rob (with length $l$ ) under the gravity acceleration $g$. Without any friction, the angle $\theta$ with the vertical is described by the following equation:

$$
d^{2} \theta / d t^{2}=-g / l \sin (\theta)
$$

This problem is often misconceived as being analytically unsolvable and therefore as requiring a numerical method to be solved. For instance, Gallant writes in a textbook that "there is no exact solution to this equation, so you have to solve it numerically" (2012, p. 70). Hiestand similarly writes that the simple pendulum equation "is nonlinear and cannot be solved in closed form though an approximate solution can be obtained for small values of $\theta$ " (Hiestand 2009 , p. 2). However, an analytical solution to the simple pendulum equation is available (Belendez et al. 2007, p. 647), and therefore numerical methods are in principle not required to solve the problem.

How can we explain that the analytical solution to the simple pendulum equation is not much recognized in the scientific literature while the simple

et al. 2001, p. 452). We point out that the $\mathrm{N}$-body problem with $N=2$ is an exception though, as, in this case, there are conserved quantities enough like energy, linear momentum and angular momentum to make this change of variables, and give a liouvillian solution. In this case, the system is said "integrable". 
pendulum is a very well known model in physics? We suggest the following reason: the analytical solution to the simple pendulum requires sophisticated mathematics, excessively sophisticated given the simplicity of the system. The analytical solution to the simple pendulum equation is indeed a closed-form solution expressed with special functions, which are the complete elliptic integral of the first kind $K(m)$, the incomplete elliptic integral of the first kind $u(\phi ; m)$, and the Jacobi elliptic function $s n(u ; m)$ with:

$$
\begin{aligned}
& K(m)=\int_{0}^{1} \frac{d z}{\sqrt{\left(1-z^{2}\right)\left(1-m z^{2}\right)}} \\
& u(\phi ; m)=\int_{0}^{\phi} \frac{d z}{\sqrt{\left(1-z^{2}\right)\left(1-m z^{2}\right)}} \\
& \operatorname{sn}(u ; m)=\sin (\phi)
\end{aligned}
$$

It seems to be preferable for scientists to use numerical methods instead of the exact analytical solution in order to study the behavior of the simple pendulum system. In order to see this, let us introduce the analytical solution to the simple pendulum equation. The analytical solution of the simple pendulum is the following closed-form solution based on special functions with initial conditions $\theta(0)=\theta_{0}$ and $\dot{\theta}(0)=0$ :

$$
\theta(t)=2 \arcsin \left\{\sin \left(\theta_{0} / 2\right) \operatorname{sn}\left[K\left(\sin ^{2}\left(\theta_{0} / 2\right)\right)-\omega_{0} t ; \sin ^{2}\left(\theta_{0} / 2\right)\right]\right\}
$$

Unlike the solution to the N-body problem, the solution of the simple pendulum equation can be used to make quantitative predictions. In particular, it can be used to get quantitative predictions about the motion of the pendulum. As shown in Figure 2, the analytical solution can indeed be computed over time from initial conditions. Thus, contrary to the case of the N-body

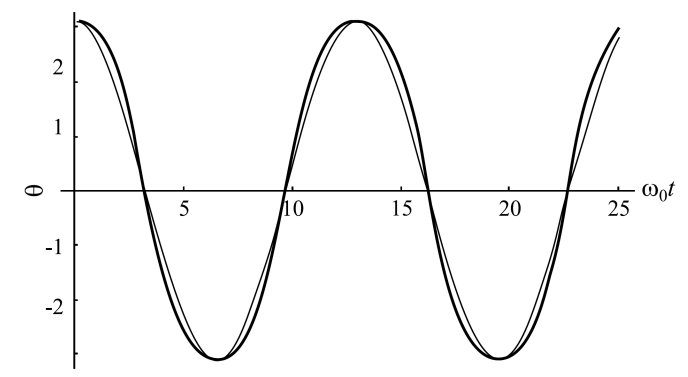

Fig. 2 The solution of the simple pendulum for initial conditions $\theta_{0}=0.9 \pi$ and $\dot{\theta}_{0}=0$ (bold line) computed with the software Mathematica. The thin line corresponds to a solution of the equation when $\theta_{0}$ is sufficiently small (Belendez et al. 2007, p. 647).

problem, one can derive accurate quantitative predictions from the analytical solution to the simple pendulum. However numerical methods are used instead 
for the purpose of making quantitative predictions. To explain this, we suggest that the analytical solution is considered by scientists as too complex for the problem at stake. The system of the simple pendulum is already a simple and elementary model. The model is highly idealized since, for example, it does not take into account air friction and represents the weight as a mass point. So, there is no point to use a very sophisticated computational tool for having exact solutions to already very idealized equations. Here, approximate numerical results are considered as satisfying. Thus, in our view, when a model is highly idealized, its analytical solution is expected to not require excessively sophisticated mathematics for the purpose of making quantitative predictions. If it is not the case, numerical methods are preferred, at least, if scientists considered them as more convenient to make quantitative predictions.

The reason for choosing numerical methods (when the analytical solution is available) can be generalized insofar as numerical errors generated by numerical methods are small enough. This is based on an assessment of numerical errors compared to modelling and experimental errors. This way of assessing numerical errors has been promoted by Fillion and Corless when they write that numerical errors "should be analyzable in the same terms as modelling and experimental errors. By that we mean that if truncation, discretization, and roundoff errors are small compared to modelling and experimental error, then for all we know, our approximate numerical answer can be the right one" $(2014$, p. 1459). It is not meant that, in such cases, scientists are doomed to make only qualitative predictions. Quantitative predictions are often (if not always) made from mathematical models which are idealized representations of target systems. We only stress that, as far as the order of magnitude of computational errors - e.g $10^{-7}$ - is smaller than the one of modelling and experimental errors - e. g. $10^{-4}$ - computational errors can be neglected. Thus, in such cases, one can give up on exactness of the analytical solution for the simplicity of the computational tool. In other words, the ease of making quantitative predictions with a numerical method (involving small numerical errors) prevails over the exactness of the analytical solution of the equation (which represents a highly idealized systems and thus involves large modelling errors).

The case of the simple pendulum contrasts in the scientific literature with the case of another mechanical system: the harmonic oscillator system. Unlike the analytical solution of the simple pendulum, the analytical solution of the harmonic oscillator is well known and well used. Our explanation of this difference derives from the previous one: both the model of the harmonic oscillator and its analytical solution are simple. The harmonic oscillator system is highly idealized and its analytical solution requires only elementary and well-known functions, i.e. the sine function.

The Lotka-Volterra model is another example that illustrates that an analytical solution must not be too complicated. The Lotka-Volterra model describes the idealized dynamics of prey and predator populations, e.g fishes and sharks, or hares and lynx. It is built on simple hypotheses about population growth and predation (cf. Murray 2002, p. 79-80). The system of model 
equations is then:

$$
d N / d t=N(a-b P) \quad \text { and } \quad d P / d t=-P(c-d N)
$$

where $N(t)$ and $P(t)$ are the respective numbers of prey and predators at time $t ; a, b, c$ and $d$ are positive constants. The constant $a$ is the growth rate of the prey population; $b$ is the rate of the prey eaten by predators; $c$ is the death rate of predators; and $d$ is the rate of eaten prey that allows for the birth of predators. ${ }^{10}$

Like the case of the simple pendulum, the analytical solution to the LotkaVolterra model is not often used although it is available (Dutt 1976; Evans and Findley 1999) ${ }^{11}$ :

$$
N(t)=\frac{1}{d}(c \omega(t)+\dot{\omega}) \quad \text { and } \quad P(t)=\frac{1}{b}(a \omega(t)-\dot{\omega})
$$

with

$$
t=t_{0}+\int_{\omega_{0}}^{\omega} d \omega^{\prime} c\left(e^{\rho}-\omega^{\prime}\right)^{-1}
$$

where $e^{\rho}$ satisfies a particular algebraic equation (Evans and Findley 1999, p. 182).

Equations (7) have thus an analytical solution, which however cannot be expressed as an elementary solution since equation (9) is not reducible to elementary functions. Therefore this solution has a complicated form while the model is highly idealized. By contrast, it is relatively easy to explore the dynamics numerically. Thus numerical methods could be preferred. For instance, the simplest forward Euler method (see Section 2) can be used to solve both equations (7) and thus to make quantitative predictions about the rate of populations and to show that the population trajectories oscillate. Here again exactness is sacrificed for ease of getting quantitative predictions.

As we will now see, there is another reason that makes numerical methods preferable to analytical solutions: analytical methods do not offer a systematic approach for solving equations of different kinds as numerical methods do.

\section{Analytical methods as non-generic approaches for solving equations}

It may be more convenient for scientists to use numerical methods for another reason. Solving equations analytically seems to require specific mathematical

\footnotetext{
10 First, in the absence of any predation, the prey grows unboundedly in a Malthusian way; this is the $a N$ term. Second, the effect of the predation is to reduce the prey growth rate by the term $b$ and by the number of predators; this is the $-b N P$ term. Third, the prey contribute to the predators' growth; this is the $d N P$ term. Fourth, in the absence of any prey to eat, the predators die following an exponential decay, that is, the $-c P$ term.

11 In (Evans and Findley 1999, p. 181-182), the equations of the Lotka-Volterra model are slightly different and written in function of the variables $x_{1}(t)$ and $x_{2}(t)$. We get the equations (7) with the mathematical transformations $N(t)=(b / d) x_{1}(t)$ and $P(t)=x_{2}(t)$.
} 
techniques depending on the equation at stake. On the contrary, numerical methods are more generic techniques for solving differential equations. The same numerical method can be used to solve very different kinds of differential equations. This property is very important in the context of scientific modelling that requires modifying the equations by adding new parameters and variables or, for example, by changing the power of an exponent.

Therefore, even if scientists know or could know the analytical solutions to the equations, the use of numerical methods could be more convenient. This is suggested in a quantum mechanics textbook:

The solution of differential equations constitutes an entire subdiscipline of mathematics. Unfortunately each different potential substituted into the Schrödinger equation typically yields a different problem, requiring a different method of solution. No single method suffices for all potentials. [...] In contrast to analytic methods, the computer solution procedures for one-dimensional potentials can be standardized. (French and Taylor, p. 174)

According to French and Taylor, solving equations analytically requires specific mathematical techniques depending on the equation at stake. For example, the mathematical techniques to solve linear differential equations are not the same as to solve non-linear equations. Similarly the way to solve first order equations is not the same as to solve second order differential equations. Solving equations analytically is sensitive to small modifications in the equations: a small change in an analytically solved equation may lead to an analytically unsolvable equation. For instance, the solution to the equation of the harmonic oscillator $d^{2} x / d t^{2}-\omega x=0$ is an elementary function. However, if the constant $\omega$ is replaced by a linear function of the time $\omega(t)=t$, the equation is not solvable with liouvillian functions (and consequently not solvable with elementary functions) but, as we have seen, with the Airy special function.

On the other hand, numerical methods are generic techniques for solving differential equations. The same numerical method can be used to solve very different kinds of equations. For example, the Runge-Kutta methods can be used to solve first-order differential equations as well as second-order and more generally n-order differential equations. Similarly, they can be used to solve linear as well as non-linear differential equations. Therefore, the same numerical methods can be used to solve an equation or a modified version of it. For example, as French and Taylor emphasize, the same numerical method can be used to solve Schrödinger's equation with different potential functions.

We suggest here a possible explanation why numerical methods are generic methods to solve differential equations: the mathematical conditions required to solve differential equations with numerical methods are weak. Therefore, the family of numerically solvable differential equations is large. Let us take again the case of the forward Euler method applied for solving the family of differential equations $d x / d t=f(x, t)$. As we have seen, this means that the following recursive algorithm $x_{k+1}=f\left(x_{k}, t_{k}\right) h+x_{k}$ is used to build the series $\left(x_{k}\right)$. The more kinds of functions for $f(x, t)$ are admitted, the more generic the 
Euler method is. At first glance, $f(x, t)$ can be polynomial functions, trigonometric functions, and so on. Yet it turns out that the required conditions for $f(x, t)$ to use the Euler numerical method are weak: Euler method is convergent if $f(x, t)$ is lipschitzian in $x$ (Stoer and Bulisch 2002, p. 478-480; Süli and Mayers 2003, p. 322). This means that there is a positive constant $M$ such as $\left|f\left(x_{1}, t\right)-f\left(x_{2}, t\right)\right| \leq M\left|x_{1}-x_{2}\right|$, which corresponds to a criterion of regularly. Many functions satisfy this criterion and the Euler function can thus be applied in principle to all of them. ${ }^{12}$ In addition, since problems of differential equations of order $n$ can be rewritten with $n$ differential equations of order 1 , one can suppose that the generality of numerical method for greater orders can be explained the same way.

One can thus understand why, even if analytical solutions can be known, scientists may choose numerical methods. They are generic techniques, which is a particularly useful way to find solutions in the context of scientific modelling. Now that we have presented three main cases where numerical methods are preferred to analytical solutions, let us examine what they tell us about the often presumed superiority of analytical solutions.

\section{Ease of making quantitative predictions}

It is commonly accepted that analytical solutions are superior to numerical methods. Sometimes the reasons for such a claim concern the mathematical elegance of analytical solutions or the talent a mathematician needs to have in order to find them. In this paper, we focus rather on objective criteria. Analytical solutions can be useful in providing information about several properties of the target system like its symmetry, periodicity, linearity or non-linearity. In addition, analytical solutions are general solutions. As functions of variables, they are solutions for potentially many cases depending on the numerical values assigned to the variables. These criteria may play an explanatory role, and therefore be important for scientific theorizing. But they are not relevant for assessing analytical solutions and numerical methods in making quantitative predictions because this task concerns specific cases. In particular, we agree with Humphreys' claim that the loss of generality in numerical methods "is not a great loss, for applications are ordinarily to specific cases" (Humphreys 2004, p. 65).

We have thus suggested that exactness is an important criterion. But, on the other hand, we have shown that scientists might prefer numerical methods to analytical solutions when making quantitative predictions is easier with numerical methods. We have found three main instances where this is the case: First, some analytical solutions have no practical use when they can only be expressed as infinite series and have slow convergence. Second, analytical solutions are sometimes an excessively sophisticated mathematical machinery

\footnotetext{
12 We emphasize how close are the conditions that guarantee that the numerical Euler method is convergent, and the conditions that guarantee that there exists a unique solution to a differential equation $d x / d t=f(x, t)$ with an initial condition (Picard-Lindelöf theorem).
} 
for the problem at stake. Third, analytical methods do not offer a generic approach for solving equations of different kinds.

In this way, we suggest that there is another criterion for assessing value of mathematical solutions. This criterion might be called ease of making quantitative predictions. Because, as we have seen, this criterion sometimes prevails over exactness of solutions, we claim that analytical solutions cannot be considered superior to numerical methods.

One could think that ease of making quantitative predictions is a lesser criterion than exactness in that it is a practical criterion and therefore cannot threaten the absolute superiority of analytical solutions. Indeed, ease of getting numerical results is important because scientists are cognitively limited agents. So long and tedious tasks - that getting numerical results from analytical solutions may involve - constrain the choice of scientists between different computational tools. But the practical aspects make a great difference as soon as we endorse the distinction between science in principle and science in practice (Humphreys 2004).

Science in principle includes the epistemic activities that an ideal agent could conduct; an ideal agent having unlimited cognitive capacities. This definition does not take into account the concrete operations that an agent with limited capacities could actually do, nor the relative difficulty of the operations required in the different epistemic activities. On the other hand, science in practice takes into account the perspective of the agent and therefore includes the epistemic activities that real agents - i.e. cognitively limited agents - can actually perform.

In the context of science in practice, ease of making quantitative predictions becomes a criterion as important as exactness of solutions. In science in practice, there seems to be no rational argument that allows one to assign a higher epistemic value to exactness than to ease of getting numerical results. Besides, while preferring ease of getting numbers to exactness is a matter of pragmatic choice, the reasons for this choice are based on the very mathematical properties of analytical solutions. The three above-studied reasons for preferring numerical methods are inherent to the mathematical forms of analytical solutions. In other words, some mathematical properties of analytical solutions (like requiring complicated special functions or exemplifying slow convergence for infinite series) make the task of making quantitative predictions difficult and sometimes impossible.

In the context of science in principle, ease of making quantitative predictions is not, by definition, a criterion for the superiority of analytical solutions. This criterion is irrelevant since agents are considered as ideal. Therefore, one could think that analytical methods are still superior to numerical methods. However science in principle is an ideal picture that is far from what scientists can really predict or describe. Furthermore, the frontier between science in principle and science in practice is sometimes thin. For instance, slow convergence of infinite series might still be a case that threatens the superiority of analytical solutions in science in principle (e.g the N-body problem). All 
of this suggests that, in the context of science in practice, the superiority of analytical solutions over numerical methods has to be mitigated.

\section{Conclusion}

We argued that numerical methods are sometimes preferable to analytical ones for the purpose of making quantitative predictions because: (i) some analytical solutions make numerical applications difficult or impossible; (ii) analytical solutions are sometimes an excessively sophisticated mathematical machinery for the problem at stake; (iii) analytical methods do not offer a systematic approach for solving equations of different kinds like numerical methods do.

These three reasons result from the more general fact that analytical solutions are exact but do not always facilitate getting numerical results. Numerical methods are thus sometimes better in making quantitative predictions. Even though opting for ease of getting numerical results over exactness of solutions is a matter of pragmatic choice, this choice is nevertheless meaningful. It suggests that, in the context of science in practice, the often presumed superiority of analytical solutions must be mitigated.

\section{Acknowledgements}

\section{References}

1. Babelon, O., Bernard, D. \& Talon, M. (2003). Introduction to classical integrable systems, Cambridge ; New York : Cambridge University Press.

2. Barberousse, A., Franceschelli, S. \& Imbert, C. (2009). Computer simulations as experiments, Synthese, 169 (3), p. 557-574.

3. Barberousse, A. \& Imbert, C. (2014). Recurring Models and Sensitivity to Computational Constraints, The Monist, 97 (3), p. 259-279.

4. Batterman, R. W. (2007). On the Specialness of Special Functions (The Nonrandom Effusions of the Divine Mathematician), The British Journal for the Philosophy of Science 58 (2), p. 263-286.

5. Belendez, A., Pascual, C., Mendez, D.I. Belendez, T. \& Neipp, C. (2007). Exact solution for the nonlinear pendulum, Revista Brasileira de Ensino de Fisica, 29 (4), p. 645-648.

6. Borwein, J. \& Crandall, R. (2013). Closed Forms: What They Are and Why We Care, Notices of the American Mathematical Society, 60 (1), p. 50-65.

7. Corless, R. M. and Fillion, N. (2014). A Graduate Introduction to Numerical Methods, Springer

8. Diacu, F. (1996). The Solution of the N-boby Problem, The Mathematical Intelligencer 18 (3), p. $66-70$

9. Dutt, R. (1976). Application of Hamilton-Jacobi Theory to the Lotka-Volterra Oscillator Bulletin of Mathematical Biology, 38, p. 459-465.

10. Einstein, T.L (2003). Applications of Ideas from Random Matrix Theory to Step Distributions on "Misoriented" Surfaces, Ann. Henri Poincaré 4, Suppl. 2, p. 811-824.

11. Evans, C.M. \& Findley, G.L. (1999). Analytic solutions to a family of LotkaVolterra related differential equations, Journal of Mathematical Chemistry, 25, p. 181-189.

12. Fillion, N. \& Corless, R.M. (2014). On the Epistemological Analysis of Modeling and Computational Error in the Mathematical Sciences, Synthese, 191, p. 1451-1467.

13. Fillion, N. \& Bangu, S. (2015). Numerical Methods, Complexity and Epistemic Hierarchies, Philosophy of Science. forthcoming.

14. Forsythe, G. E. (1970). Pitfalls in computation, or why a math book isn't enough. The American Mathematical Monthly, 77(9), p. 931-956. 
15. French, A. P. \& Taylor, E. F. (1998). An Introduction to Quantum Physics, Cheltenham, United Kingdom: Stanley Thomas.

16. Gallant, J. (2012). Doing Physics with Scientific Notebook, Wiley.

17. Goldstein, H., Poole, C. \& Safko, J. (2001). Classical mechanics, Addison-Wesley Publishing Company.

18. Goriely, A. (2001). Integrability and nonintegrability of dynamical systems, Advanced series in nonlinear dynamics volume 19, World Scientific.

19. Hartmann, S. (1996). The world as a process - Simulations in the natural and social sciences. In R. U. M. Hegselmann \& K. Troitzsch (Eds.), Modelling and simulation in the social sciences from the philosophy of science point of view (p. 77-100). Dordrecht: Kluwer.

20. Hairer, E., Nørsett, S.P. \& Wanner, G. (1992). Solving Ordinary Differential Equations I : Nonstiff problems, Springer.

21. Hiestand, J. W. (2009). Numerical Methods with VBA Programming, John \& Bartlett Publishers.

22. Henkel, Malte (2001). Sur la solution de Sundman du problme des trois corps, Philosophia Scientiae, 5(2), p. 161-184.

23. Humphreys, P. (2004). Extending Ourselves: Computational Science, Empiricism, and Scientific Method, New-York : Oxford University Press.

24. Humphreys, P. (2009). The philosophical novelty of computer simulation methods. Synthese, $169(3)$, p. $615-626$.

25. Masoliver, J. \& Ros, A. (2011). Integrability and chaos : the classical uncertainty, European Journal of Physics, 32, p. 431-458.

26. Murray, J. (2002). Mathematical biology. An Introduction, vol. 1, Springer.

27. Morrison, M. (2009). Models, measurement and computer simulation: The changing face of experimentation. Philosophical Studies, 143, p. 33-57.

28. Ortega, J. M. (1992). Numerical Analysis, Ed. SIAM.

29. Singer, M. F. (1990). Formal Solutions of Differential Equations, Journal of Symbolic Computation, 10, p. 59-94.

30. Stern, A. \& Desbrun, M. (2008). Discrete Geometric Mechanics for Variational Time Integrators. In Discrete Differential Geometry : An Applied Introduction, Siggraph 2006 Course Notes, chap. 15.

31. Stoer, J. \& Bulisch, R. (2002). Introduction to Numerical Analysis, Texts in Applied Mathematics 12, Springer.

32. Süli, E. \& Mayers, D.F. (2003). An Introduction to Numerical Analysis, Cambridge: Cambridge University Press.

33. Sundman, K. (1907). Recherches sur le problème des trios corps, Acta Societatis Scientiarum Fennicae, 34 (6).

34. Sundman, K. (1909). Nouvelles recherches sur le problème des trois corps, Acta Societatis Scientiarum Fennicae, 35 (9)

35. Wang, Q.-D. (1991). The Global Solution of the N-Body Problem, Celestial Mechanics and Dynamics Astronomy, 50, p. 73-88.

36. Zoladek, H. (1998). The Extented Monodromy Group and Liouvillian First Intergrals, Journal of Dynamical and Control Systems, 4 (1) , p. 1-28. 